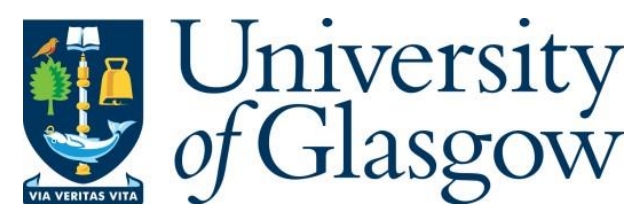

Streicker, D. G. and Gilbert, A. T. (2020) Contextualizing bats as viral reservoirs. Science, 370(6513), pp. 172-173.

There may be differences between this version and the published version. You are advised to consult the publisher's version if you wish to cite from it.

http://eprints.gla.ac.uk/223443/

Deposited on: 24 September 2020

Enlighten - Research publications by members of the University of Glasgow http://eprints.gla.ac.uk 


\section{Contextualizing bats as viral reservoirs}

\section{Preventing zoonotic emergence from bats requires integrative research}

By Daniel G. Streicker ${ }^{1,2}$ and Amy T. Gilbert ${ }^{3}$

Coronavirus disease 2019 (COVID-19) is the latest in a distressing tally of viral infections, including Ebola, Nipah, rabies, severe acute respiratory syndrome (SARS), and Middle East respiratory syndrome (MERS), which have evolutionary origins or epidemiological associations with bats. This seeming preponderance of zoonoses has propelled bats from biomedical obscurity to the forefront of global health. Immunological traits, potentially shaped by bat life history, have been proposed to allow bats to control viruses differently from other animals. However, incomplete baselines for broader comparisons across vertebrates and extensive immunological variation among bat species casts uncertainty on their uniqueness as viral reservoirs. Moreover, common perceptions that bats asymptomatically harbor viruses more often than other animals and that their viruses are more diverse or pose systematically heightened zoonotic risk remain unresolved. The search for answers may inspire new approaches to manage disease threats to human and animal health.

Bats (order Chiroptera) comprise $\sim 1400$ species which split from the remaining members of the Scrotifera (carnivores, pangolins, cetaceans, odd and even toed ungulates) over 60 million years ago during the Late Cretaceous. The capacity for true flight, unique to bats among mammals, opened diverse trophic niches, making bats key providers of global ecosystem services, including insect pest control, seed dispersal, and pollination of agricultural plants. Flight also introduced physiological challenges that transformed bat life history. For example, aerial transport of young restricts litter sizes to one or two pups annually across most species. The need for multiple bouts of reproduction to maximize fitness therefore favored longevity, hypothesized to be mediated by adaptations to suppress tumors and limit inflammation from DNA damage (1).

\footnotetext{
${ }^{1}$ Institute of Biodiversity, Animal Health and Comparative Medicine, College of Medical, Veterinary and Life Sciences, University of Glasgow, Glasgow, UK. ${ }^{2}$ MRCUniversity of Glasgow Centre for Virus Research, Glasgow, UK. ${ }^{3}$ United States Department of Agriculture, Animal and Plant Health Inspection Service, National Wildlife Research Center, Fort Collins, CO, USA. Email: daniel.streicker@glasgow.ac.uk, amy.t.gilbert@usda.gov
}

Perhaps serendipitously, these mechanisms also limit virus-induced inflammation, potentially explaining why viruses including Marburg virus, SARS-coronavirus (SARS-CoV) and MERS-CoV are thought to cause subclinical infections in the presumed natural bat hosts (Rousettus aegyptiacus for Marburg virus and Rhinolophus spp. for both CoVs), but immunopathology in other vertebrates. Over evolutionary timescales, limited inflammatory responses in bats, together with high population densities and gregarious social behaviors that may facilitate virus transmission, may have selected for viruses that cause severe disease in incidental hosts that lack analogous defenses.

Peculiarities in bat immune systems that plausibly alter viral interactions are increasingly recognized (2). Whether bats are exceptional in this respect is unclear since knowledge of vertebrate immunity largely derives from inbred mice or immortalized cells, which diverge substantially from wild relatives. Fortunately, the rise in genome sequencing has provided crucial phylogenetic context to the evolutionary origins of bat immunity while facilitating comparisons to diverse non-model species (3). For example, comparative transcriptomics showed distinct aspects of innate immunity in bats (Myotis lucifugus and Pteropus vampyrus), but also in eight other mammalian and avian species (4). By characterizing distinct antiviral features across taxa, efforts to contextualize bat immunity might reveal discoveries that inspire new strategies to prevent and treat viral zoonoses in humans and animals.

Heightened interest in bat-associated viral zoonoses has also revealed striking immunological variation among species. For example, black flying foxes $(P$. alecto) have an unusually contracted interferon (IFN)- $\alpha$ locus (genes encoding early components of the innate immune response) and cells that constitutively express IFN- $\alpha$, thereby inducing antiviral activity (5). However, other bats have expanded IFN- $\alpha$ loci and lack constitutive IFN- $\alpha$ (6). Similarly, bat species with elevated constitutive antiviral defenses may do so via differing gene expression pathways (4), and the antiviral APOBEC gene family has undergone bat linage specific expansions or duplication (3). This im- plies that some of the unusual antiviral defenses in bats arose independently after the evolution of flight. Analogous to other vertebrates, divergent immunological repertoires among bat species may reflect alternative responses to biogeographic variation in viral assemblages and environmental conditions. Identifying the eco-evolutionary determinants and host range of antiviral defenses might help identify unreported reservoirs of zoonoses but requires expanding research beyond the relatively few bat species already known to transmit zoonoses.

Whether features of bat immunology predictably translate into functionally unique antiviral strategies is unresolved. For example, the popular notion that bats subclinically tolerate virus infections is supported by experimental infections of bats with high-profile viruses, including Marburg virus, Ebola virus and MERS-CoV. Conversely, other viruses that may be lethal to humans, including lyssaviruses, Tacaribe virus, and Lloviu virus (a distant relative of Ebola virus with unknown human pathogenicity) are also apparently lethal to bats, including putative reservoir hosts. Sublethal effects of viruses on wild bats are largely undetectable because individuallevel, longitudinal monitoring is only possible in species that live in relatively small groups and are reliably recaptured. Individual heterogeneities that alter infection outcomes in humans and other animals, such as age, sex, social hierarchies, and past and contemporaneous infections remain virtually unexplored in bats. Given limited evidence from wild populations, meta-analyses of experimental infections might test whether bats systematically manifest less clinical disease than other host groups. Other taxa that are subclinically infected with some zoonoses, such as rodents (e.g., Lassa virus, hantaviruses) and birds (e.g., eastern equine encephalitis virus, West Nile virus) provide relevant contrasts.

Whether bat viruses are disproportionately zoonotic is an outstanding global health conundrum. A meta-analysis of 2,805 host-virus interactions showed bats are more likely than other mammals to be infected by viruses that infect humans (7). Yet, when analyses are restricted to hosts that 
are believed to be important for natural transmission cycles, viral richness among bats was unexceptional and they contributed approximately the number of zoonoses expected for the number of species in this group (8). Thus, evolutionarily conserved traits of bats seem unlikely to produce viruses with inflated zoonotic capability. Heightened susceptibility or surveillance may explain why bats appear to incidentally host a relatively large number of zoonoses.

Once introduced into the human population, are bat viruses exceptionally dangerous? One meta-analysis found higher case fatality ratios (CFRs) and lower human-tohuman transmissibility among bat viruses; however, the extent that these patterns generalize among bat viruses was uncertain (9). The rabies-causing lyssaviruses, which comprise $\sim 50 \%$ of zoonotic viruses recognized from bats (8), exemplify high CFRs and low transmissibility among humans, but being lethal across all mammals do not fit the emerging paradigm of tolerance in bats contrasted with virulence in humans. Deviations such as SARS-CoV-2 (low CFR and high transmissibility) and the ebolaviruses (moderate CFR and transmissibility) highlight further complexity to explore.

If the virulence of bat viruses is systematically elevated, mathematical models fit to in vitro experiments provide a possible explanation: accelerated viral propagation with limited cellular morbidity might favor chronic subclinical infections in bats, but acute infections in other hosts (10). Although the prediction that bat viruses that cause short-lived, lethal infections in humans infect bats chronically remains unconfirmed in vivo, the short timeframes and small sample sizes of most experiments make detecting reactivation of latent viral infections in bats unlikely. Ultimately, virulence is an emergent property of host and virus interactions. As such, determining whether differences among species arise from virus-specific phenomena within bats, ill-fitted responses of naïve immune systems, or generalizable viral tolerance mechanisms may require profiling immunological responses and within-host dynamics across diverse viruses and host species.

Beyond contextualizing the distinctiveness of bat reservoirs, fundamental and applied research should also tackle the realworld complexity underlying viral zoonotic emergence (see the figure). A first step may be to identify how intrinsic traits of bats and extrinsic factors interact to govern viral transmission, community assembly and zoonotic emergence. For example, spatiallyreplicated metagenomic sequencing in vampire bats (Desmodus rotundus) found no evidence that larger colonies sustain more viruses, but revealed elevational gradients and age biases in viral diversity (11). In flying foxes (Pteropus spp.), longitudinal monitoring showed pulsed shedding of multiple paramyxoviruses (a virus family associated with several emerging zoonoses), potentially arising from physiological stress induced by acute food shortages (12). Understanding virus co-infection and community dynamics may also reveal recombination opportunities that potentially enable emergence. Anticipating how anthropogenic perturbations such as land use change and persecution of bats for consumption, trade, fear, or misguided efforts at disease control will precipitate emergence is a greater challenge. These actions can alter both viral transmission among bats and the frequency of interspecies contacts (including with intermediate hosts) but are conceptually underdeveloped and rarely tested empirically.

Integrating understanding of the zoonotic process across biomedical, population and ecosystem scales may enable bold new approaches to prevent zoonotic emergence by reducing virus circulation in bat reservoirs. Knowledge of bat genomics and immunity opens the door for using genetic technologies like CRISPR to engineer viral resistance in wild bats, analogous to ongoing efforts to control Lyme disease in wild mice (13). Historical barriers to delivering vaccines at sufficient scales to alter viral dynamics in wild bat populations are also diminishing. The relative ease of metagenomic sequencing enables rapid discovery of naturally occurring, innocuous, and speciesspecific bat viruses that might be engineered into transmissible vaccines targeting zoonoses in wild bats. This theoretically appealing idea has empirical precedents from efforts to vaccinate wild rabbits against myxomatosis and rabbit hemorrhagic disease (14). Advantageously, the traits expected to facilitate virus transmission in some bats, such as gregariousness and flight, could similarly support live vaccine dissemination, while naturally slow demographic turnover would help to maintain vaccine-induced population-level immunity, allowing less frequent interventions (15).

Such potentially transformative strategies require rigorous investigation into efficacy, safety and ecological impacts as well as overcoming barriers to societal acceptance. Viruses such as rabies virus, Marburg virus, and henipaviruses, where bat reservoirs are known, host and viral genomes are available, and transmission to humans and/or animals occurs with measurable frequency can serve as tractable and important models to evaluate and refine candidate interventions.

At present, viral emergence from bats is largely unpredictable and unpreventable. Solutions will require qualitative and quantitative expansions over current practice in biomedical and epidemiological bat research, which only rarely consider heterogeneities among individuals, populations and species. This variability is powerful because it can reveal the ultimate drivers and phenotypic importance of bat-virus interactions as well as whether they generalize in ways that might aid surveillance or management of zoonotic threats. Given the costs of the COVID-19 pandemic, the need for such an ambitious research agenda is more evident now than ever.

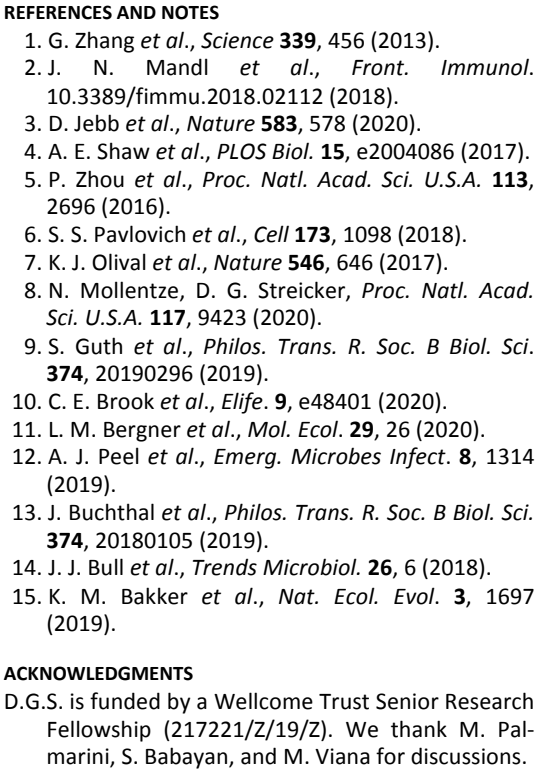

Title: Dimensions of zoonotic emergence Bat viruses emerge through currently unpredictable interactions of evolutionary and ecological forces. Intrinsic features of bat immune systems have been shaped by bat life history and past viral interactions. Anthropogenic perturbations may alter host-virus interactions at the individual or population levels, while breaking down historical barriers between species, culminating in viral emergence. 